\title{
Using Co-Design to Examine How Children Conceptualize Intelligent Interfaces
}

\author{
Julia Woodward', Zari McFadden ${ }^{3 \dagger}$, Nicole Shiver ${ }^{1}$, Amir Ben-hayon ${ }^{1}$, Jason C. Yip ${ }^{2}$, Lisa Anthony \\ Department of CISE, University of Florida, Gainesville, Florida, USA ${ }^{1}$ \\ University of Washington - The Information School, Seattle, Washington, USA ${ }^{2}$ \\ Spelman College, Atlanta, Georgia, $\mathrm{USA}^{3}$ \\ julia.woodward@ufl.edu,jcyip@uw.edu, lanthony@cise.ufl.edu \\ + Work conducted while a summer intern at the University of Florida.
}

a)

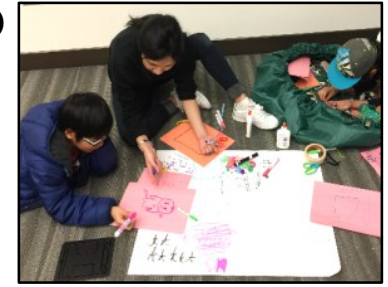

b)

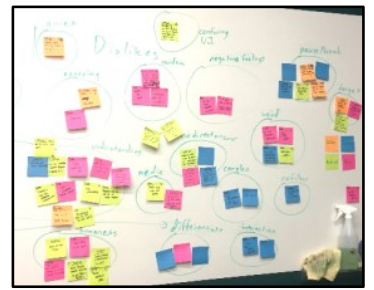

c)

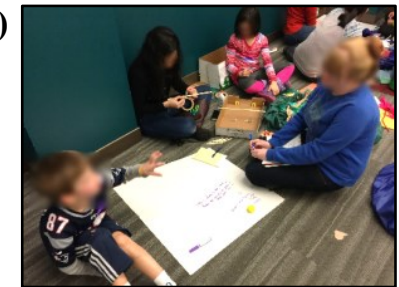

d)

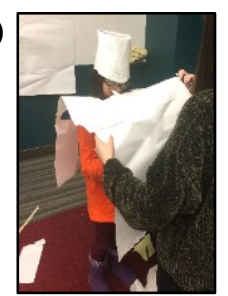

Figure 1: Participatory design sessions with the children. (a) Design Session 1: Bags of Stuff and Big Paper. (b) Design Session 2: Stickies. (c) Design Session 3: Bags of Stuff and Big Paper. (d) Design Session 4: Big Props.

\section{ABSTRACT}

Prior work has shown that intelligent user interfaces (IUIs) that use modalities such as speech, gesture, and writing pose challenges for children due to their developing cognitive and motor skills. Research has focused on improving recognition and accuracy by accommodating children's specific interaction behaviors. Understanding children's expectations of IUIs is also important to decrease the impact of recognition errors that occur. To understand children's conceptual model of IUIs, we completed four consecutive participatory design sessions on designing IUIs with an emphasis on error detection and correction. We found that, while children think of interactive systems in terms of both user input and behavior and system output and behavior, they also propose ideas that require advanced system intelligence, e.g., context and conversation. Our work contributes new understanding of how children conceptualize IUIs and new methods for error detection and correction, and will inform the design of future IUIs for children to improve their experience.

\section{Author Keywords}

Intelligent user interfaces; children; conceptual model; error correction; participatory design; co-design; design methods.

Permission to make digital or hard copies of all or part of this work for personal or classroom use is granted without fee provided that copies are not made or distributed for profit or commercial advantage and that copies bear this notice and the full citation on the first page. Copyrights for components of this work owned by others than the author(s) must be honored. Abstracting with credit is permitted. To copy otherwise, or republish, to post on servers or to redistribute to lists, requires prior specific permission and/or a fee. Request permissions from Permissions@acm.org.

CHI 2018, April 21-26, 2018, Montreal, QC, Canada (C) 2018 Copyright is held by the owner/author(s). Publication rights licensed to ACM. ACM 978-1-4503-5620-6/18/04 ...\$15.00

https://doi.org/10.1145/3173574.3174149

\section{ACM Classification Keywords}

H.5.m. Information interfaces and presentation (e.g., HCI): Miscellaneous.

\section{INTRODUCTION}

Children are increasingly presented with natural interaction technologies, including intelligent user interface (IUI) modalities such as speech, gesture, and writing [29,30,36]. In this paper, we use the term IUIs to refer to interfaces in which the system tries to interpret the user's intent [50]. The design of educational technology and games geared toward younger users $[10,40,48]$ is made more challenging by the fact that children's interaction behaviors and expectations have been shown consistently in childcomputer interaction literature to be different from those of adults $[1-3,5,25,26,37,38,49,53,60]$. Prior work has shown that IUIs that use modalities such as speech, gesture, and writing pose challenges for children due to their developing cognitive and motor skills [42,51]. For example, gesture recognition algorithms do not perform as well for children $[2,3,60]$, mainly due to their motor skills development affecting their writing ability [45].

Prior research has focused on improving recognition and accuracy by accommodating children's specific interaction behaviors $[1,2,36-38,46,49,52,60]$. Researchers have characterized children's interactions on smartphones and other devices by collecting and analyzing touch and gesture data $[1,2,37,49,52,60]$. For instance, Shaw and Anthony [49] examined children's gestures and highlighted features that can be used to create new recognition algorithms and gesture sets that are more tailored toward children. Woodward et al. [60] examined how interface complexity affects children's interactions by collecting touch and gesture data, and provided design guidelines based on quantitative analysis. Although these approaches are 
effective in analyzing children's interactions to reduce recognition errors, designing IUIs to better support children's conceptual expectations could help decrease the impact of recognition errors when they do occur [4]. In addition, recognition is only one aspect of making IUIs usable for children. Other important aspects of IUIs include what affordances and limitations children believe exist in these systems, what children's thoughts are about errors (e.g., to what do they attribute errors), and other facets of user perception. Understanding children's perceptions and expectations of IUIs matters as much as accuracy and recognition because usability errors can occur based on mismatches between users' and designers' expectations [39]. This perspective focuses on how a person and a machine collaborate together, rather than just making command-based corrections. We believe that, to understand how a child and an IUI can collaborate together, it is necessary to develop a conceptual model [28] of how a child thinks about an IUI.

Developing a conceptual model about children's understanding of IUIs would be challenging with conventional methods (e.g., observations, usability studies) because of the wide spectrum of IUIs that are possible. For example, IUIs can include touchscreens, voice-activated assistants, gestures and motion, and other UI paradigms. Also, for young children, direct interviews can be difficult because of (1) fatigue and time constraints; (2) power dynamics between adults and children; (3) confusion about interview questions; and (4) trouble verbalizing their mental models $[35,71]$. Therefore, in our work we use participatory design (PD, co-design), specifically the Cooperative Inquiry method [12,34], since it has been shown to elicit richer ideas from children when compared to interviews $[7,11]$.

To understand how children conceptualize IUIs, we conducted four consecutive PD sessions with an intergenerational co-design group that consisted of seven children (ages 7 to 12), as seen in Figure 1. The sessions concentrated on designing IUIs with an emphasis on error detection and correction. We used a different design activity for each session that focused on eliciting how children conceptualize IUIs and new approaches to error correction that make sense to them. We aimed to answer three research questions:

(1) How do children think about and understand IUIs?

(2) Can getting direct input from children through PD elicit novel design recommendations for error detection and correction?

(3) Do different PD techniques elicit different parts of the conceptual model?

We analyzed video and audio recordings from the design sessions by creating an affinity diagram [8] of the children's utterances. We used this diagram to construct a conceptual model of children's understanding of IUIs. We found that, while children think of interactive systems in terms of both user input and behavior and system output and behavior, they also propose ideas that require advanced system intelligence, e.g., social context and conversation. We also analyzed the types of data that were elicited from each PD session to understand which PD techniques more effectively influenced which parts of the model.

The contributions of our work are as follows: (1) new insights into how children conceptualize IUIs; (2) new design recommendations for error detection and correction for IUIs for children; and (3) new understanding of how different PD techniques reveal different parts of a complex conceptual model. In fact, to date our work is the first to use PD techniques to create a model of user interaction. Our goal here is to demonstrate that co-design can be used to develop children's conceptual model of IUIs; future work could validate this model with follow-up studies. Our work will inform the design of IUIs for children that improve their experience by supporting their expectations and decreasing the impact of recognition error recovery.

\section{RELATED WORK}

We focus our review of prior work on three major categories: (1) children's interactions with IUIs; (2) children's conceptual understanding of technology; and (3) participatory design (PD) methods.

\section{Children's Interactions with IUls}

Most previous work on the topic of children's interactions with IUIs, including the speech, gesture, and writing modalities, has focused on understanding children's interaction behaviors and recognition algorithm accuracy [1,2,36-38,45,46,49,52,60]. Anthony et al. [2] examined touch and gesture interactions by children (ages 7 to 16) and adults on a mobile device. They offered design guidelines, such as training age-specific recognizers to improve children's gesture recognition accuracy. McKnight and Cassidy [37] studied the interactions of 7- to 10-yearolds with small-screen mobile devices to create a set of ten guidelines for designers. For example, they recommended that a stylus should be considered for children when fine accuracy is required, and software solutions may help in increasing finger-interaction accuracy. Lovato and Piper [36] examined how young children use voice input systems by analyzing YouTube videos of children using Siri and conducting an online survey of parents. They identified three primary ways children use voice input systems: exploration (e.g., trying to understand the system), information seeking (e.g., asking questions), and function (e.g., trying to operate the device). The authors also noted that recognition of children's speech is an open challenge since they observed low accuracy in the videos. They suggested trying to detect social speech patterns (e.g., pausing or yelling) to improve recognition of children's speech. Instead of focusing on understanding the user's interaction behaviors in order to improve recognition, we aimed to understand how children conceptualize IUIs. This work will inform the design of IUIs that meet children's expectations and decrease the impact of recognition errors. 


\section{Children's Understanding of Technology}

Although not explicitly in the domain of IUIs, some prior work has examined how children understand and define "technology." Jarvis and Rennie [27] outlined a five-stage model of how children develop their understanding of "technology," broadly defined, through analysis of child drawings and one-on-one interviews. The five stages are: No Model, Embryonic Ideas (ideas are unlikely to be immediately established), Single Explanation (idea is held consistently), Multiple Explanations (ideas are inconsistently applied and vary in conviction), and Development of a Generalized Concept. Wang and Komlodi [59] used interviews and focus groups to also examine how children define "technology." They analyzed children's verbal definitions and found that all the definitions included aspects associated with the context of use such as being entertaining, complex, and useful.

Related to IUIs, prior work has examined how understanding children's parasocial relationships (onesided emotional relationships) with media characters can aid in designing intelligent software agents [9,21]. Brunick et al. [9] surveyed prior research to examine how components of parasocial relationships (e.g., attachment) may assist in the design of intelligent agents as educational tools. The authors suggested that intelligent agents should have the ability to generate parasocial interactions, such as conversational timing and response personalization. Gray et al. [21] described their experiences designing for parasocial relationships at Sesame Workshop and emphasized that factors such as social realism and personification should be considered when designing an intelligent agent for children. They also identified new factors unique to physical interaction, such as what modality and media to use (e.g., audio, interactive screen, or physical embodiment).

Prior work has examined children's conceptual models in science learning contexts, with the goal of developing instructional interventions that "fit" the children's model $[20,55]$. In contrast to this work on models of natural phenomena, there is little work on children's mental models of complex technical systems. Also, our model aims to inform the design of IUIs for children, instead of developing instruction. In our work we focused on capturing the children's expectations and ideas rather than explicitly eliciting knowledge. Researchers in HCI have also developed conceptual models of children's use of certain interactive technology. For example, Druin et al. [16] and Foss et al. [18,19] both developed conceptual models of Internet search roles of children (ages 7 to 11 $[16,18]$ ) and teens (ages 14 to 17 [19]) by conducting interviews and observing search tasks.

In contrast to prior work, we are the first to focus specifically on how children's conceptual model of IUIs can inform the design of the interactions in general (rather than only for intelligent agents), and the first to use PD techniques to dig in more deeply than interviews or observations can allow. By knowing how children perceive and relate to IUIs, we can better anticipate possible errors.

\section{Participatory Design Methods and Techniques}

For this study, we conducted four consecutive Cooperative Inquiry PD sessions [12,13,34,62], with a group of seven children. PD is a method of design that brings users and designers together to co-create new technologies [31]. Cooperative Inquiry is a type of PD method that emphasizes close partnerships with children $[13,62]$. Since the 1990's, researchers in child-computer interaction have incorporated PD techniques like Cooperative Inquiry into creating children's technologies [7,11-13,34,58,62]. Creative and fun PD techniques, such as KidReporter [7] and Mission From Mars [11], have been shown to elicit inventive and expressive ideas. For our PD techniques, we chose to use Big Paper [22,58], Bags of Stuff [12,58], Stickies [12,58], and Big Props [58] (more details on these techniques will be discussed under Design Sessions). We apply these creative PD techniques in our study to elicit children's conceptual model of IUIs.

\section{METHOD}

Our PD sessions focused on designing IUIs with an emphasis on error detection and correction. We chose to use the Cooperative Inquiry method with an existing co-design group for two reasons: the children (1) already work closely with adults and are more likely to express themselves; and (2) are already knowledgeable on multiple PD techniques. We conducted four 90-minute sessions across a span of two weeks, with two design sessions a week. Each design session consisted of a design activity chosen for its potential to explore different aspects of the design space of children's conceptual model of IUIs:

Day 1: Handwriting Game: design a touchscreen game that supports handwriting (Bags of Stuff, Big Paper).

Day 2: Speech Agents: use and evaluate four different speech agents (Stickies).

Day 3: User-Initiated Error Correction: design ways a user could initiate and respond to error detection and correction in an IUI (Bags of Stuff, Big Paper).

Day 4: System-Initiated Error Correction: act out how the system could initiate and respond to error detection and correction in an IUI (Big Props).

\section{Participants}

An intergenerational co-design group, consisting of both adult design researchers and child participants, called KidsTeam $U W$, participated in the four design sessions.

\begin{tabular}{llll} 
Name & Age & Gender & Ethnicity \\
\hline Chris & 7 & Male & Hispanic \\
Aiyana & 8 & Female & Asian/Black \\
Matt & 9 & Male & White \\
Sierra & 9 & Female & Asian/White \\
Krista & 10 & Female & Asian/White \\
Mason & 10 & Male & Native/Asian \\
Keegan & 12 & Male & Asian
\end{tabular}

Table 1. Demographic characteristics of our child participants. All names are pseudonyms. 
KidsTeam $U W$ includes seven children ages 7 to $12[\mathrm{M}=$ 9.3, $\mathrm{SD}=1.6]$ (see Table 1). This age group is consistent with previous work on Cooperative Inquiry $[7,12,14,22,57,62]$. These children are old enough to codesign, but are still developing cognitive and motor skills relevant to the use of IUIs $[2,22,42,51]$. The children's technological experience is consistent with findings from USA national surveys [72]. All of the child participants have Internet at home and some of them carry mobile devices (e.g., smartphones) to co-design sessions. The adults were six undergraduate students, four graduate students, and one professor [ages $\mathrm{M}=25.3, \mathrm{SD}=5.6$ ]. Our protocol was approved by both UF and UW's institutional review boards, and all names in this paper are pseudonyms.

\section{Design Sessions}

Each design session consisted of snack time (15 minutes), circle time (15 minutes), design time (45 minutes), and discussion time (15 minutes). Snack time and circle time were warm-up activities and social time. In circle time, we asked a "question of the day" to get adults and children started. During design time, the team broke into smaller, intergenerational design groups to complete the day's design activity using PD techniques [12,22,58]. During discussion, each group would present their finished designs and the whole team would reflect and discuss common themes. During circle time and discussion, we included several direct questions in a group interview format. We chose each design technique for each day based on what design artifacts we wanted the children to produce [58]. We organized the sessions to scaffold the children's thought processes on IUIs by first introducing the concept of IUIs, having the children use different speech agents, and then examining user-initiated and system-initiated error detection and correction (Table 2). We video and audio recorded each session, and a rotating facilitator moved the camera throughout the session to focus on different codesign groups. Each day, we photographed design artifacts and collected notes.

\section{Design Session 1 - Handwriting Game (DS 1)}

On the first day, we introduced the children to the concept of IUIs and different modalities. The design activity was to design a handwriting touchscreen game. The only design constraint was that the game had to recognize writing. We used two different PD techniques: Bags of Stuff $[12,58]$ and Big Paper [22,58]. Bags of Stuff is a low-tech prototyping technique (Figure 1a). Large bags are filled with craft materials, i.e., construction paper and markers. Each design group uses the materials to create a low-fidelity prototype. Big Paper is a form of paper prototyping in which each design group has a large piece of paper to draw on, usually from an easel pad. The large size of the paper allows space for collaboration.

\section{Design Session 2 - Speech Agents (DS 2)}

On the second day, we elicited information regarding how children think about and use different speech agents. We used four different speech agents: Microsoft Cortana [64],
Amazon Echo [65], Apple Siri [66], and Google Android Assistant [67]. The design groups rotated using the different speech agents and evaluated them using the Stickies PD technique [12,58] (Figure 1b). In Stickies, each design group uses sticky notes to write down likes, dislikes, and design ideas for the technological device they are evaluating. The speech agents were live and responded to the children's statements, which were completely unconstrained by us.

Design Session 3 - User-Initiated Error Correction (DS 3) On the third day, we introduced the concept of error detection and correction. The design activity was to design technology that would respond to the user when the user informs the system that there is an error in the system's understanding or the user's input. We focused on two questions: how do you want to tell the system that there is an error? and how do you want the system to respond? We used the PD techniques from DS 1 (Bags of Stuff and Big Paper, Figure 1c) for this design activity.

Design Session 4 - System-Initiated Error Correction (DS 4) On the final day, we continued exploring error detection and correction, but from the system-initiated perspective. During the design activity, the children took on the role of the IUI and acted out how the system would initiate and respond to error detection and correction. Errors could be in either the user's input or the system's understanding. We used the Big Props PD technique [58], a form of scenariobased design [47] in which each design group uses props (we used easel pads and craft materials) to act out a scenario (Figure 1d). The children in each group played the role of the system, and the adults played the users.

\section{Data Analysis}

The first author transcribed the videos from the design sessions. There was a total of 279 minutes of video data (not including snack time), which resulted in a total of 534 utterances (308 from children). Due to the camera being moved throughout each design session to focus on different co-design groups, we transcribed individual conversations

\begin{tabular}{|c|c|c|}
\hline $\begin{array}{l}\text { Design } \\
\text { Session }\end{array}$ & $\begin{array}{l}\text { Circle Time } \\
\text { Question }\end{array}$ & Design Prompt \\
\hline 1 & $\begin{array}{l}\text { What's your favorite } \\
\text { touchscreen app? }\end{array}$ & $\begin{array}{l}\text { Design a handwriting } \\
\text { touchscreen game. }\end{array}$ \\
\hline 2 & $\begin{array}{l}\text { What question would } \\
\text { you ask a computer? }\end{array}$ & $\begin{array}{l}\text { Complete likes, dislikes, and } \\
\text { design ideas for Cortana, } \\
\text { Alexa, Siri, and Google } \\
\text { Assistant. }\end{array}$ \\
\hline 3 & $\begin{array}{l}\text { Name an error you got } \\
\text { from a smartphone or } \\
\text { computer. }\end{array}$ & $\begin{array}{l}\text { Design technology to respond } \\
\text { to us when we say there is an } \\
\text { error. }\end{array}$ \\
\hline 4 & $\begin{array}{l}\text { How do you want } \\
\text { technology to be better } \\
\text { designed for you? }\end{array}$ & $\begin{array}{l}\text { Act out the computer role. Act } \\
\text { back to the adult, when they } \\
\text { make a mistake or when you } \\
\text { do not understand them, how } \\
\text { you want to respond back to } \\
\text { the user. }\end{array}$ \\
\hline
\end{tabular}

Table 2. Prompts for Design Sessions. 


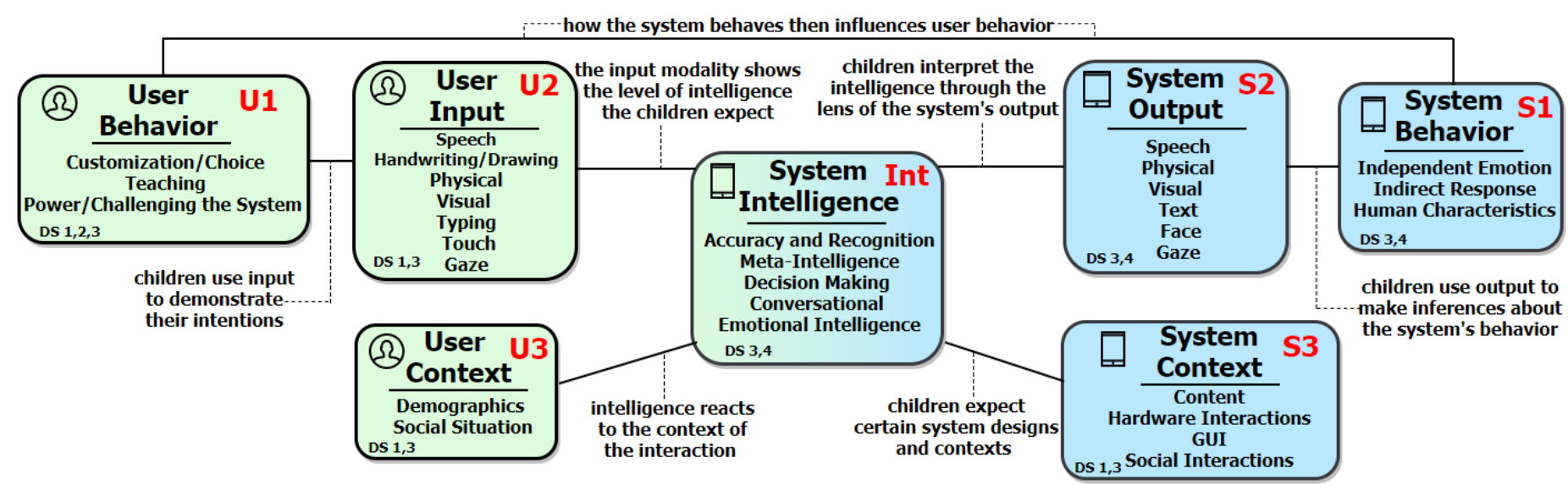

Figure 2. A conceptual model based on children's understanding of intelligent interfaces and the errors they might encounter.

as done in previous work in PD [62], instead of transcribing the design session in its entirety.

We analyzed the data using affinity diagramming, which is a method for organizing large-scale qualitative data [8]. Affinity diagramming is a bottom-up, inductive approach; we iteratively defined, refined, and combined individual data points (notes) into themes. We constructed our affinity diagram using RealtimeBoard, an online whiteboard for remote team collaboration [68]. We visually represented each statement from the transcription with a digital sticky note. The whole team iteratively organized the notes into themes over the course of several group discussions. We identified 30 individual themes, which we combined into seven major themes. The boxes in Figure 2 are the major themes; the sub-lists in each box are the individual themes organized by prevalence of notes from our affinity diagram. We also note the design sessions that aided in identifying each theme (e.g., DS 1, 2, 3, or 4). We used the major themes from the affinity diagram along with how the children spoke about and used these concepts in their designs to identify the links and relationships between the themes, which became our conceptual model.

\section{FINDINGS: THE CONCEPTUAL MODEL}

We identified seven (7) major high-level themes through affinity diagraming: User Behavior, System Behavior, User Input, System Output, User Context, System Context, and System Intelligence (Figure 2).

We present an overview of the conceptual model here and then discuss each individual theme in the model, and how it is supported by our data. First, we describe how the themes are related. In our data, the children talked about using input to demonstrate their own intentions to the system (U2 to $U 1$ in Figure 2). When the children suggested a specific input modality, it was related to the level of intelligence that they expected the system to demonstrate (U2 to Int). The children assumed certain system contexts and domains would be supported by IUIs (Int to $S 3$ ), as well as that the intelligence of the system would react to the context of the interaction and the user (Int to U3). The children's designs clearly demonstrated that they were interpreting the intelligence of the system through the lens of the system's output ( $S 2$ to $I n t)$, which children used to make inferences about the internal behavior or intentions of the system $(S 2$ to $S 1$ ). Finally, there is a cyclical and dynamic connection as the system behavior that the child infers ultimately influences how the child will respond (S1 to U1). Far from focusing solely on specific features pertaining to intelligence, children designed whole interactive systems with complete feature sets. We were able to extract more detail about their conceptual model from these holistic designs than single point-solutions.

\section{Themes}

We will now discuss each of the major themes and subthemes of the conceptual model in detail, with examples from our co-design sessions.

User Behavior (U1)

During the design activities, we observed the children interacting with their designs and existing speech agents or describing how to use their designs, which provided insight into the user's behavior (DS 1, 2, and 3). In the children's designs, the users held most of the power and control through Customization, Teaching the system, and Challenging the System. In the children's conceptual model, an intelligent system must learn from the user.

Customization and Choice (U1). A major theme apparent throughout the designs was user customization and choice. The children designed the ability to change colors, avatars, and the current mood of the system. In DS 4, Sierra (Table 1) had a button which the user could press to change the mood (affect) of the system (e.g., sassy, sweet):

Adult: "So hit that button to change your mood?"

Sierra: "Yeah, that's the button." (child as system)

The children's designs frequently used avatars and characters; for example, in one design the user could draw their own avatar to customize it: "And then you have an avatar, you can draw it." [Krista, DS 3]. In the children's 
conceptual model, an intelligent system should support a wide range of user customization, whether through visual elements or being able to change the system's characteristics. An IUI should be tailored to the user's preferences to create a customized interaction experience.

Teaching (U1). When they were explicitly focusing on error detection and correction (DS 3 and 4), the children included the idea of the user teaching the system. For example, the user can provide images: "Show a picture of how it actually should be [...] Show how to make it right." [Matt]. Also, the user can tell the system to "learn", and the system will correct itself: "So when there is an error there is a little character that pops up, and [...] you can choose an unknown character and it grows when you press 'learn'." [Krista]. This theme requires advanced system intelligence: to be aware of what the user is intending and correct future mistakes. As for user behavior, this contributes to the concept of the user having control and taking on a constructive role in the interaction.

Power and Challenging the System (U1). A frequent theme in the children's design suggestions was the way they asserted power and challenged the system. For instance, when talking about the system, Aiyana stated, "Well you can treat it however way you want." [DS 1]. The children also challenged the system through giving "attitude" and questioning its intelligence: "Are you even alive?" [Aiyana, DS 2]. In DS 2, Matt questioned the system's intelligence by asking: "When is the next time you will understand what I am saying?" This shows that the children feel comfortable challenging the intelligence of the system, and that they expect a high level of recognition.

\section{System Behavior (S1)}

We identified themes in how the children conceptualized internal system behaviors from the designs they suggested (DS 1) and from how they acted out the system initiating error detection and correction (DS 4). Several individual themes emerged: Emotion, Indirect Response, and Human Characteristics. All of the individual themes show that children expect human-like behavior from the system.

Independent Emotion (S1). As part of Intelligence, the system should respond with appropriate emotion, either positive or negative, depending on the context of the situation. In some of the children's designs, the system responded with emotion without any input or provocation from the user. For example, during DS 4, Krista responded back to the user (adult) by refusing to answer:

Adult: "Do you want to correct me?"

Krista: "No, I am too bored." (child as system)

In the children's conceptual model, an IUI could respond with independent emotion, which they think of as natural behavior related to intelligence.

Indirect Response (S1). During DS 4, children commonly responded to the user (adult) with indirect responses:

Adult: "What should I do tomorrow?"
Chris: "You can go to a new place"

Adult: "What new place?"

Chris: "Why don't you choose?"

Therefore, in children's mental model, the system does not always need to directly address a question or statement from the user. This design implication might stem from children's frustrations at current IUIs not always understanding them. This view on system behavior contrasts with the children's model of user behavior which included Power and Challenging the System. In the children's conceptual model, both the user and the system can challenge each other, implying a more balanced control.

Human Characteristics (S1). While designing, the children personified the system by giving it human characteristics such as visual facial features. For example, in one design idea (DS 4), the user has the ability to give the system different faces, which will change the system's characteristics:

Adult: "Oh you can change the face as well?"

Krista: "Mhmm (confirmation)” (child as system)

This theme captures the idea of the IUI behaving in a more human-like manner. The IUI exhibits human-like behavior through emotion, but also has human facial characteristics. In the children's mental model, an intelligent system goes beyond acting human-like by also visually exhibiting physical human characteristics.

User Input (U2)

The children in our study integrated a comprehensive list of input modalities into their designs. Speech and handwriting or drawing were the most common forms of input, probably due to our design constraints (e.g., children had to include handwriting in DS 1). However, the children also included other input modalities such as hardware (e.g., pen or gavel), wearables (e.g., smart bracelets), and visual input (e.g., images and changing elements on the screen). As an example of visual input, Chris stated, "Write really big and you can change the size of it." [DS 1]. Chris' design included a feature to change the size of handwriting on the screen by pressing different arrow buttons. Many of the modalities the children proposed to use would require some type of recognition-based intelligence, e.g., speech or handwriting recognition: "You can write on it and talk to it." [Aiyana, DS 1]. The proliferation of different input modalities shows that the children expected a high level of system flexibility and that systems should be able to support whatever modality they wanted to use.

System Output (S2)

The children in our study designed for a comprehensive number of output modalities. System speech and physical output (e.g., throwing colorful cotton pom-poms) were the most common. In DS 4, the children incorporated physical items such as craft pom-poms and confetti as output to relay the system's level of understanding. For example, "So if I didn't understand your questions I would throw these (craft 
pom-poms)." [Chris, DS 4]. While the materials we provided likely influenced their ideas, it is more important to note the affect and emotion that the children conveyed. For instance, the children incorporated physical items to relay positive emotion: "You wrote your name and then you get a disco ball (craft pom-poms come out and "Celebrate" song is playing)." [Mason, DS 1]. Common output modalities from the children were visual animations, colors, and characters. The children interpreted the intelligence of the system through its output and made inferences about what it meant about the internal behavior of the system.

\section{User Context (U3)}

While co-designing, the children in our study incorporated the context of the interaction between the user and the system. Based on the types of suggestions in this theme, we divided User Context into two individual themes: Demographics and Social Situation.

Demographics (U3). While designing, the children talked about the age and gender of the user. This illustrates that in the children's conceptual model, technology should be designed for individual users instead of being designed the same for everyone. For instance, Krista thought about how younger children might not be able to use a standard pen and designed a thicker pen: "This pen is for toddlers because they don't have a good grip." [DS 1].

Social Situation (U3). The children also expected the system to understand the social context of the interaction. Their mental model is that an IUI should go beyond accurately recognizing input to understanding the social context of the input to yield better results for the user. In DS 1, Sierra designed the system to understand that "Happy Birthday" would be a special event: "If you write something special in the text like Happy Birthday, it will send some sparks flying or something."

\section{System Context (S3)}

In their designs, children emphasized certain contexts (e.g., content domains). The children incorporated Content, Hardware Interactions, Visual Elements (GUI), and Social Interactions into their designs. We grouped these related individual themes into the major theme of System Context, based both on how they were used by the children and what the children's intentions were when they included them.

Content (S3). The children considered content as a basis for their design, which determined many of the other features of the intelligent interface. Content was an important aspect to the children, whether it was designing a handwriting game or designing approaches for error correction. For example, in DS 1, Chris created a space game in which the user could handwrite the names of the planets, as well as battle aliens: "Touch it and it goes away (the aliens)." When designing approaches to user-initiated error correction (DS 3), they designed thematic characters that would appear to inform the user of an error: "Bird says there is an error." [Chris].
Hardware Interactions (S3). The children designed multiple hardware-based input modalities (e.g., pen) to communicate with the IUI. They repeatedly designed ways to use hardware to provide feedback and interact with the system (e.g., using a gavel to tap the screen to say "yes" or "no"). Krista designed a wearable bracelet that the user could use to change the color of text appearing on the screen: "You can use the bracelet to change the color (by touching the pen to it). And then you can choose your age and write your name (with the pen)." [DS 1]. Also, the children thought about having a "special" pen to be used for certain interactions (e.g., a pen only for errors). In the children's minds, an intelligent system should be able to communicate with physical hardware to help create a mixed interaction (e.g., physical and digital) depending on context.

GUI (S3). A common theme in the children's designs was the inclusion of visual screen elements (e.g., color, animations, and effects). Sierra designed a painting handwriting game for DS 1 that utilized visual effects: "And then if I put my paintbrush in here, then boom it's all sparkly and this one is a rainbow." The children used visual elements for effects, as well as for providing feedback. During DS 3, Aiyana designed visual elements (e.g., shapes and colors) to provide feedback to the user about the degree of the error: "Let's say the error is here and if it gets worse it will change from blue to orange." In the children's conceptual model, an IUI should use visual screen elements as a way to communicate with the user, through feedback on error correction or illustrating the social context of an interaction (e.g., Happy Birthday).

Social Interactions (S3). Socialization is an important factor to the children when using IUIs; they wanted to be able to communicate and share with their friends within the games they designed (DS 1). Many existing applications have a socializing element (e.g., chatting with friends $[69,70]$ ), which might have influenced the children's designs. However, in many of their designs, the children also wanted the system to interact with them and communicate as a friend. In DS 1, Aiyana designed a stuffed toy which included a screen to handwrite messages to friends: "It's like a pet and a little friend, it talks to you, and asks you to play a game." This relates to the theme of System Intelligence: an IUI should be able to maintain a social conversation.

\section{System Intelligence (Int)}

Throughout the design activities, it was clear that children expect an advanced level of intelligence in IUIs. In the children's designs, the system accurately recognized the user's input and intentions, and responded in an intelligent manner including both conversation and emotion. Also, the system should be able to detect when it does not understand the user. The children have high expectations for the capabilities of the system. We separated the theme of intelligence into individual themes: Accuracy and Recognition, Meta-Intelligence, Decision Making, 
Conversational, Emotional Intelligence, and Other. The Other category consists of sub-themes within Intelligence that had a small number of sticky notes that could not be combined with other themes. For example, children suggested that the system should have Simple Knowledge, such as understanding the time or day, and being able to count steps: "[...] it counts your steps and if you take 100 steps you win the game." [Aiyana, DS 1].

Accuracy and Recognition (Int). Through examining the children's designs and statements, we found that the children expect a competent level of input recognition, whether the modality is speech, gesture, or writing. Also, the children believe that the system should be aware of the user's abilities and be able to recognize different languages and user handwriting, without any additional input from the user. For example: "People who are better skilled in writing with different languages, it should recognize any widely spoken language." [Keegan, DS 1]. There was no sense from the children that they might have to configure or train the system in any way. The children identified that a current limitation of existing systems is that they don't always recognize their input: "Sometimes it doesn't detect you." [Chris, DS 1]. An IUI will have to correctly recognize the user's input as a way to know how to intelligently respond (e.g., making Decisions, maintaining a Conversation, or responding with Emotion).

Meta-Intelligence (Int). One theme that manifested from the design activity in which the children acted as the system (DS 4) is that the system should be aware of when it is confused and doesn't understand the user. When acting as the system, the children would frequently ask for clarification or inform the user that they did not understand instead of guessing: "I did not understand that." (child as the system) [Chris]. This behavior could be interpreted as the children's mental model for how a system should respond in such as case. The children complained when the system incorrectly recognized them: "I talked to Siri and said, 'Where is the nearest place you can shut up?' and she said, 'What do you want me to search up?'” [Matt, DS 3]. According to the children, the system should also know when it is right or wrong: "And when I get it right, I'll throw that (throws craft pom-poms)." (child as the system) [Chris]. This kind of response requires advanced intelligence, as the system needs to be aware of its own understanding; current systems can approximate this through recognition confidence scoring techniques [24]. This theme contrasts with current IUI approaches, which often try to hide the system's uncertain understanding through guessing or auto-correction. While the children expect a high level of recognition and accuracy, they also want the system to seek clarification when the user's input is not understood.

Decision Making (Int). In the children's designs, the system is able to recognize the user's input and intentions so it can make decisions regarding error correction and how to respond. For example, the system should be able to offer different possibilities based on the user's input:

Adult: "Show me sushi."

Krista: "This, this, or this?" (child as the system providing visual options) [DS 4]

In Aiyana's design (previously mentioned in System Context: GUI), the system provided different colors based on the severity of an error: "Let's say the error is here and if it gets worse it will change from blue to orange." [DS 3]. The children viewed the system's ability to make these kinds of decisions, without input from the user, as an intelligent aspect necessary in an IUI.

Conversational (Int). While designing, the children consistently conceptualized the system as having the ability to maintain a natural conversation. In their designs, the system communicates with the user and understands enough of the context of the situation needed to start and maintain a conversation. This echoes recommendations from prior work to include parasocial interactions in intelligent agents $[9,21]$. For instance, during DS 4, this exchange occurred during a pizza ordering scenario:

Adult: "I like pizza"

Sierra: "Which is your favorite pizza place?" (child as system)

For the system to respond in this way is a step beyond input recognition; the system is determining a response without an explicit question or command from the user. In the children's conceptual model, an IUI should be able to respond with human-like characteristics such as the ability to maintain a conversation.

Emotional Intelligence (Int). One common theme throughout the children's designs was presence of emotion. According to the children, the system should have the capacity to elicit, recognize, and accurately respond to emotion (which facilitates parasocial interactions [9,21]). The children viewed emotion as both input and output; the user can express emotion, and the system can respond with emotion. In DS 1, Aiyana designed a stuffed toy (the system) that would respond with emotion by moving its eyes: "So you see this stuffed animal, the way his eyes move shows how he feels, it's like a digital little friend." In the children's conceptual model, an IUI must recognize the user's emotions (e.g., frustration) through the user's input and provide output that includes an appropriate emotion. Understanding emotion can be difficult even for humans [41], but this type of intelligence was a fundamental part of many of the children's designs (DS 1, 4).

\section{DISCUSSION}

We can see from the depth of the conceptual model that the children in our study exhibited evidence that their mental models are in the most mature stage (Developing a Generalized Concept) of Jarvis and Rennie's [27] five-stage model of how children understand "technology." Based on the conceptual model we developed, the children in our 
study have an understanding of interactive systems as a whole, as well as specific aspects of what makes a system intelligent, including both input (user input and behavior) and output (system output and behavior). We focus our discussion on (a) comparing our conceptual model produced by PD to existing design recommendations from studies focusing on recognition and accuracy; (b) comparing the different PD techniques to understand how they reveal children's perspectives of IUIs; and (c) suggesting new design recommendations for error detection and correction based on our conceptual model.

\section{Comparing Conceptual Model to Existing Solutions}

As mentioned, previous work in child-computer interaction with IUIs has focused on improving recognition and accuracy to accommodate children's specific interaction behaviors $\quad[1,2,36-38,44-46,49,52,60]$. For example, Woodward et al. [60] suggests using more examples when training gesture recognizers for younger children. However, we believe using recognition and accuracy to mitigate errors is only one part of the solution. Given that general-purpose recognition in modalities such as speech and gesture is likely to never reach $100 \%$ accuracy $[3,44,49,60]$, it is important to consider how to prevent the impact of the inevitable recognition errors. While past design recommendations help decrease recognition errors, they do not consider children's expectations and abilities in context. Our work illuminates children's expectations of IUIs when errors occur. For example, based on our conceptual model, we know that the children expect a competent level of input recognition, whether the modality is speech, gesture, or writing (e.g., Accuracy and Recognition). However, when errors do occur, the IUI should be Conversational, and also admit when it does not understand the user's input or intention, seeking clarification from the user (e.g., Meta-Intelligence). The user can take on a Social and constructive role (e.g., Teaching) in the error correction process. Through considering children's conceptual model of IUIs along with design recommendations for improving accuracy and recognition, we can design technology that will decrease the impact of recognition errors when they occur.

\section{Comparison of Participatory Design Techniques}

An important contribution of our study is a reflection on how our PD methods helped us create our conceptual model, as the first work to do so. Since each of the design sessions contributed to different portions of the conceptual model, it is clear that using a single PD technique or session would not have been sufficient. DS 1 (Bags of Stuff for error correction) allowed us to elicit more ideas focused on System Context (e.g., content, hardware), which would be useful for designing specific applications for children. DS 2 (Stickies) allowed us to observe the children interacting with speech agents and provided insights into what the children thought about existing IUIs. This information could be used to evaluate existing systems and provide insight into user behavior. However, by themselves DS 1 and DS 2 did not elicit as many deep ideas that illuminated the children's conceptual model as DS 3 and DS 4.

Instead, these first two sessions allowed us to set up the context for the more complex design sessions. Using Bags of Stuff and Big Paper for user-initiated error correction (DS 3) allowed us to extract new design recommendations for error detection and correction based on the children's own designs. While they were the same PD techniques as DS 1 , the scope of the activity was more complex. Having the children act as the system with Big Props (DS 4) proved to be the most beneficial in understanding how children conceptualize IUIs. It produced the most engagement with the activities and identified complex themes (e.g., Intelligence), and it allowed us to see how the children expect an IUI-based system to act. We recommend that $\mathrm{HCI}$ researchers interested in developing complex models using PD techniques start with sessions allowing participants to become familiar with the context, and structure later sessions to focus on more elaborate interactions.

\section{Using Interviews and Observation}

In our PD sessions, we incorporated group interview questions into the circle time and discussion phases of the design sessions. For example, in DS 4, we asked the children how they would define the term "smart technology." We received very simple answers such as "Cool" [Krista] and "Smart" [Sierra], which contrasts with the complex themes about smart system interfaces that we extracted from the PD activities (e.g., Meta-Intelligence). Although interviews are a more scalable approach than PD and allow collecting data from more participants, our data shows that interview questions did not produce as insightful and complex data as the PD techniques; most likely because PD techniques allow the children to concretely express abstract ideas [14]. In addition, we thought that observing the children interacting with current intelligent technology (DS 2) would help us understand their mental models. We categorized the children's questions and statements while interacting with the different speech agents (DS 2) and observed that they spent most of the time seeking information or media (e.g., "play a song"), findings that echo Lovato and Piper's observational study of YouTube videos [36]. We did find that children asked the speech agents personal questions (e.g., "When is your birthday?" [Sierra]), which supports our model's theme that children desire IUI behaviors with human-like characteristics.

Overall, when considering complex technological interactions for children, such as how they conceptualize IUIs, our experience demonstrates that conducting interviews and observations do not generate data as rich as PD techniques do. Quality of data from interviews may depend on the types and depth of questions (e.g., "What makes technology smart?"). Observing children while they use technology may produce data that includes what they like or dislike, but we did not find that it revealed much about their conceptual model. When considering how 
children conceptualize complex technical systems, PD techniques are useful in producing data with depth and complexity. While it may not be feasible to reproduce these methods with large groups of children to validate the conceptual model, our in-depth rich engagement with children in PD sessions is an important first step. For instance, interviews with larger groups of children, probing on specific aspects of the model, could see how the model generalizes to other children besides our co-designers.

\section{Design Recommendations}

Based on our conceptual model, we suggest new design recommendations for error detection and correction in speech, gesture, and writing. Previous literature, as well as commercial systems that use these modalities, approach error detection and correction in various ways: (a) underlining [6,54], (b) auto-correcting [32,63], (c) highlighting [32], and (d) suggesting alternatives [17,32]. In most of the cases, the user is responsible for correcting the error. In the following sections, we discuss new ideas we developed based on the children's conceptual model.

\section{Conversational Interactions}

A key insight from our conceptual model is that the children consistently conceptualized the IUI being able to maintain a Conversation. In the children's conceptual model, the IUI should respond in a human-like manner (e.g., Human Characteristics), which contrasts with existing techniques of system-initiated error detection and correction (e.g., auto-correcting). For the IUI to maintain a conversation, it must accurately recognize the user's input and intentions (e.g., Accuracy and Recognition), and then make decisions regarding error correction and how to respond (e.g., Decision Making). The children also expected the IUI to understand the social context (e.g., Social Situation) of the interaction. According to the children, the interaction with the IUI should be freeflowing, rather than command-based. The IUI should also have the capacity to elicit, recognize, and respond to Emotion. Conversation and emotion enable the children to have parasocial interactions with the IUI, which can facilitate learning [9]. We suggest that designers incorporate conversational prompts into error correction techniques to gain insight into the user's intentions.

\section{"I Don't Know"}

A surprising finding from our conceptual model was that children wanted the IUI to seek explicit clarification when it did not understand (e.g., Meta-Intelligence). This theme contrasts with current trends in error detection and correction, which favor the IUI trying to recover from errors on its own, through guessing or auto-correction $[32,63]$. When the children acted as the system (DS 4), they sought clarification from the user, e.g., by stating "Can you ask another thing?" [Chris]. While the children expected a high level of recognition and accuracy (e.g., Accuracy and Recognition), they also want the IUI to seek clarification. This finding points to a potential for higher tolerance toward system errors from children (e.g., [33,44]), and echoes the human-like expectations that children have for IUIs. We suggest that designers not be shy about having the IUI say, "I don't know" or asking the child to rephrase as a method for error correction for children.

\section{Limitations and Future Work}

Our study presents a rich analysis of children's conceptual model of IUIs that will inform future research and future systems. There are some limitations to the scope of our work. First, we had seven children participate in our codesign sessions. This number may seem small, but it is similar to previous work on PD with children $[15,22,23,62]$. We view our conceptual model as a starting point to understanding how children conceptualize IUIs that should be expanded through future work. Also, this conceptual model focuses on theoretical generalization, rather than statistical generalization [61]. Our PD techniques would not be feasible with larger groups of children; therefore, we suggest refining our model with different techniques. One technique that might be useful is Warp Speed Design [43]; children are first educated about the possibilities before completing a fast-paced design session. Second, the economic, social, and technological backgrounds of the children in our study can be viewed as a limitation since they were all recruited from Seattle, Washington, USA, and had experience with IUIs and technology. For future work, children could be recruited from different backgrounds in geographically distributed co-design sessions [56]. We recommend that researchers use our co-design methods with children from different economic, social, cultural, and technological backgrounds as a comparison.

\section{CONCLUSION}

We conducted four consecutive Cooperative Inquiry design sessions with a group of seven children that focused on designing IUIs with an emphasis on error correction. We constructed a conceptual model based on children's understanding of IUIs and found that the children conceptualize IUIs as whole systems and expect advanced system intelligence. DS 4 (Big Props) elicited the richest themes, while interviewing the children and observing them interact with technology failed to elicit the same depth as the PD activities. We present new design recommendations for error correction for children that are aligned with their conceptual model. By integrating our work on understanding how children conceptualize IUIs with prior work on techniques for improving accuracy, we can design better IUI technology that is tailored towards children.

\section{ACKNOWLEDGMENTS}

We thank the children and project partners in KidsTeam UW, and Kidsteam at University of Maryland for their advice on this project. We thank Tamara Clegg for her feedback on the paper. This work was partially supported by National Science Foundation Grant Award \#IIS1433228. Opinions, findings, and conclusions or recommendations expressed in this paper are those of the authors and do not necessarily reflect these agencies' views. 


\section{REFERENCES}

1. Lisa Anthony, Quincy Brown, Jaye Nias, and Berthel Tate. 2013. Examining the need for visual feedback during gesture interaction on mobile touchscreen devices for kids. In Proceedings of the International Conference on Interaction Design and Children (IDC '2013), 157-164. https://doi.org/10.1145/2485760.2485775

2. Lisa Anthony, Quincy Brown, Jaye Nias, Berthel Tate, and Shreya Mohan. 2012. Interaction and recognition challenges in interpreting children's touch and gesture input on mobile devices. In Proceedings of the ACM Interational Conference on Interactive Tabletops and Surfaces (ITS '2012), 225-234.

https://doi.org/10.1145/2396636.2396671

3. Lisa Anthony, Quincy Brown, Berthel Tate, Jaye Nias, Robin Brewer, and Germaine Irwin. 2014. Designing smarter touch-based interfaces for educational contexts. Personal and Ubiquitous Computing 18, 6: 1471-1483. https://doi.org/10.1007/s00779-013-07499

4. Lisa Anthony, Jie Yang, and Kenneth R. Koedinger. 2012. A paradigm for handwriting-based intelligent tutors. International Journal of Human-Computer Studies 70, 11: 866-887. https://doi.org/10.1016/j.ijhcs.2012.04.003

5. Ahmed Sabbir Arif and Cristina Sylla. 2013. A comparative evaluation of touch and pen gestures for adult and child users. In Proceedings of the International Conference on Interaction Design and Children (IDC '2013), 392-395. https://doi.org/10.1145/2485760.2485804

6. Titus Barik, Jim Witschey, Brittany Johnson, and Emerson Murphy-Hill. 2014. Compiler error notifications revisited: an interaction-first approach for helping developers more effectively comprehend and resolve error notifications. In Proceedings of the International Conference on Software Engineering (ICSE'2014), 536-539. https://doi.org/10.1145/2591062.2591124

7. Mathilde Bekker, Julie Beusmans, David Keyson, and Peter Lloyd. 2003. KidReporter: a user requirements gathering technique for designing with children. Interacting with Computers 15, 2: 187-202. https://doi.org/10.1016/S0953-5438(03)00007-9

8. Hugh Beyer and Karen Holtzblatt. 1999. Contextual design. Interactions 6, 32-42.

https://doi.org/10.1145/291224.291229

9. Kaitlin L. Brunick, Marisa M. Putnam, Lauren E. McGarry, Melissa N. Richards, and Sandra L. Calvert. 2016. Children's future parasocial relationships with media characters: the age of intelligent characters. Journal of Children and Media 10, 2: 181-190. https://doi.org/10.1080/17482798.2015.1127839
10. Cynthia Chiong and Carly Shuler. Learning: Is there an app for that? Investigations of young children's usage and learning with mobile devices and apps. The Joan Ganz Cooney Center at Sesame Workshop, New York, NY. Retrieved September 12, 2017 from http://dmlcentral.net/resources/4496

11. Christian Dindler, Eva Eriksson, Ole Sejer Iversen, Andreas Lykke-Olesen, and Martin Ludvigsen. 2005. Mission from Mars: a method for exploring user requirements for children in a narrative space. In Proceedings of the International Conference on Interaction Design and Children (IDC '2005), 40-47. https://doi.org/10.1145/1109540.1109546

12. Allison Druin. 1999. Cooperative inquiry: developing new technologies for children with children. In Proceedings of the ACM SIGCHI Conference on Human Factors in Computing Systems (CHI'1999), 592-599. https://doi.org/10.1145/302979.303166

13. Allison Druin. 2002. The role of children in the design of new technology. Behaviour \& Information Technology 21, 1: 1-25. https://doi.org/10.1080/01449290110108659

14. Allison Druin, Ben Bederson, Angela Boltman, Adrian Miura, Debby Knotts-Callahan, and Mark Platt. 1999. Children as our technology design partners. In The Design of Children's Technology. Morgan Kaufmann Publishers, 51-72.

15. Allison Druin, Benjamin B. Bederson, Juan Pablo Hourcade, Lisa Sherman, Glenda Revelle, Michele Platner, and Stacy Weng. 2001. Designing a digital library for young children. In Proceedings of the Joint Conference on Digital Libraries (JCDL '2001), 398405. https://doi.org/10.1145/379437.379735

16. Allison Druin, Elizabeth Foss, Hilary Hutchinson, Evan Golub, and Leshell Hatley. 2010. Children's roles using keyword search interfaces at home. In Proceedings of the ACM SIGCHI Conference on Human Factors in Computing Systems (CHI'2010), 413-422. https://doi.org/10.1145/1753326.1753388

17. Mark D. Dunlop, Andrew Glen, Sunil Motaparti, and Sanjay Patel. 2006. AdapTex: contextually adaptive text entry for mobiles. In Proceedings of the International Conference on Human-Computer Interaction with Mobile Devices and Services (MobileHCI'2006), 265. https://doi.org/10.1145/1152215.1152277

18. Elizabeth Foss, Allison Druin, Robin Brewer, Phillip Lo, Luis Sanchez, Evan Golub, and Hilary Hutchinson. 2012. Children's search roles at home: Implications for designers, researchers, educators, and parents. Journal of the American Society for Information Science and Technology 63, 3: 558-573. https://doi.org/10.1002/asi.21700

19. Elizabeth Foss, Allison Druin, Jason Yip, Whitney 
Ford, Evan Golub, and Hilary Hutchinson. 2013. Adolescent search roles. Journal of the American Society for Information Science and Technology 64, 1: 173-189. https://doi.org/10.1002/asi.22809

20. Sören Frappart, Maartje Raijmakers, and Valérie Frède. 2014. What do children know and understand about universal gravitation? Structural and developmental aspects. Journal of Experimental Child Psychology 120: $17-38$. https://doi.org/10.1016/J.JECP.2013.11.001

21. James H. Gray, Emily Reardon, and Jennifer A. Kotler. 2017. Designing for Parasocial Relationships and Learning. In Proceedings of the International Conference on Interaction Design and Children (IDC '2017), 227-237. https://doi.org/10.1145/3078072.3079736

22. Mona Leigh Guha, Allison Druin, Gene Chipman, Jerry Alan Fails, Sante Simms, and Allison Farber. 2004. Mixing ideas: a new technique for working with young children as design partners. In Proceedings of the International Conference on Interaction Design and Children (IDC '2004), 35-42. https://doi.org/10.1145/1017833.1017838

23. Mona Leigh Guha, Allison Druin, Gene Chipman, Jerry Alan Fails, Sante Simms, and Allison Farber. 2005 . Working with young children as technology design partners. Communications of the ACM 48, 3942. https://doi.org/10.1145/1039539.1039567

24. Timothy J. Hazen, Stephanie Seneff, and Joseph Polifroni. 2002. Recognition confidence scoring and its use in speech understanding systems. Computer Speech \& Language 16, 1: 49-67.

https://doi.org/10.1006/csla.2001.0183

25. Juan Pablo Hourcade, Benjamin B. Bederson, and Allison Druin. 2004. Preschool children's use of mouse buttons. In Extended Abstracts of the ACM SIGCHI Conference on Human Factors in Computing Systems (CHI'2004), 1411-1412. https://doi.org/10.1145/985921.986077

26. Juan Pablo Hourcade, Keith B. Perry, and Aditya Sharma. 2008. PointAssist: helping four year olds point with ease. In Proceedings of the International Conference on Interaction Design and Children (IDC '2008), 202-209. https://doi.org/10.1145/1463689.1463757

27. Tina Jarvis and Léonie J. Rennie. 1998. Factors that Influence Children's Developing Perceptions of Technology. International Journal of Technology and Design Education 8, 3: 261-279. https://doi.org/10.1023/A:1008826320260

28. Jeff Johnson and Austin Henderson. 2002. Conceptual models: begin by designing what to design. Interactions 9, 25-32. https://doi.org/10.1145/503355.503366
29. Hilda K. Kabali, Matilde M. Irigoyen, Rosemary Nunez-Davis, Jennifer G. Budacki, Sweta H. Mohanty, Kristin P. Leister, and Robert L. Bonner Jr. 2015. Exposure and Use of Mobile Media Devices by Young Children. Pediatrics 136, 6: 1044-1050. https://doi.org/10.1542/peds.2015-2151

30. Cecilia Kang. 2013. Survey: For young children, mobile devices such as tablets, smartphones now a mainstay. The Washington Post, Washington DC. Retrieved September 12, 2017 from https://www.washingtonpost.com/business/technology/ survey-for-young-children-mobile-devices-such-astablets-smartphones-now-amainstay/2013/10/27/7e386f3c-3fl f-11e3-a62441d661b0bb78_story.html?

31. Finn Kensing and Jeanette Blomberg. 1998. Participatory Design: Issues and Concerns. Computer Supported Cooperative Work (CSCW) 7, 3-4: 167185. https://doi.org/10.1023/A:1008689307411

32. Andreas Komninos, Emma Nicol, and Mark D. Dunlop. 2015. Designed with Older Adults to Support Better Error Correction in SmartPhone Text Entry: The MaxieKeyboard. In Proceedings of the International Conference on Human-Computer Interaction with Mobile Devices and Services (MobileHCI'2015), 797802. https://doi.org/10.1145/2786567.2793703

33. Mary LaLomia. 1994. User acceptance of handwritten recognition accuracy. In Proceedings of the ACM SIGCHI Conference on Human Factors in Computing Systems (CHI'1994), 107-108. https://doi.org/10.1145/259963.260086

34. Mona Leigh Guha, Allison Druin, and Jerry Alan Fails. 2013. Cooperative Inquiry Revisited: Reflections of the past and guidelines for the future of intergenerational co-design. International Journal of Child-Computer Interaction 1, 1: 14-23.

https://doi.org/https://doi.org/10.1016/j.ijcci.2012.08.0 03

35. Sonia Livingstone and Dafna Lemish. 2001. Doing comparative research with children and young people. In Children and Their Changing Media Environment: A European Comparative Study, S. Livingstone and M. Bovill (eds.). Lawrence Erlbaum Associates, 31-50.

36. Silvia Lovato and Anne Marie Piper. 2015. "Siri, is this you?": Understanding young children's interactions with voice input systems. In Proceedings of the International Conference on Interaction Design and Children (IDC '2015), 335-338. https://doi.org/10.1145/2771839.2771910

37. Lorna McKnight and Brendan Cassidy. 2010. Children's Interaction with Mobile Touch-Screen Devices. International Journal of Mobile Human Computer Interaction 2, 2: 1-18. https://doi.org/10.4018/jmhci.2010040101 
38. Lorna McKnight and Daniel Fitton. 2010. Touchscreen technology for children. In Proceedings of the International Conference on Interaction Design and Children (IDC '2010), 238-241. https://doi.org/10.1145/1810543.1810580

39. Donald A. Norman. 2002. The Design of Everyday Things. Basic Books.

40. Cathleen Norris and Elliot Soloway. 2004. Envisioning the Handheld-Centric Classroom. Journal of Educational Computing Research 30, 4: 281-294. https://doi.org/10.2190/MBPJ-L35D-C4K6-AQB8

41. Maureen O'Sullivan. 2005. Emotional intelligence and deception detection: Why most people can't "read" others, but a few can. In Applications of Nonverbal Communication, Ronald E. Riggio and Robert S. Feldman (eds.). Lawrence Erlbaum Associates, 215253.

42. Jean Piaget. 1983. Piaget's Theory. In Handbook of Child Psychology, P. Mussen (ed.). Wiley \& Sons, New York, NY, USA.

43. Janet C. Read. 2009. Warp speed design: a rapid design method for use with children. In Extended Abstracts of the ACM SIGCHI Conference on Human Factors in Computing Systems (CHI'2009), 4681-4686. https://doi.org/10.1145/1520340.1520720

44. Janet C. Read, Stuart MacFarlane, and Chris Casey. 2003. "Good enough for what?": acceptance of handwriting recognition errors by child users. In Proceedings of the International Conference on Interaction Design and Children (IDC '2003), 155155. https://doi.org/10.1145/953536.953565

45. Janet Read, Stuart MacFarlane, and Chris Casey. 2001. Measuring the Usability of Text Input Methods for Children. In People and Computers XV-Interaction without Frontiers. Springer London, London, 559-572. https://doi.org/10.1007/978-1-4471-0353-0_35

46. Glenda Revelle and Emily Reardon. 2009. Designing and testing mobile interfaces for children. In Proceedings of the International Conference on Interaction Design and Children (IDC '2009), 329332. https://doi.org/10.1145/1551788.1551876

47. Mary B. Rosson and John B. Carroll. 2003. Scenariobased design. In The Human-Computer Interaction Handbook. Lawrence Erlbaum Associates, 1032-1050.

48. Mike Sharples, Inmaculada Amedillo Sanchez, Marcelo Milrad, and Giasemi Vavoula. 2009. Mobile learning: small devices, big issues. In Technology Enhanced Learning: Principles and Products, N. Balacheff, S. Ludvigsen, T. de Jong and S. Barnes (eds.). Springer, Heidelberg, 233-249.

49. Alex Shaw and Lisa Anthony. 2016. Analyzing the articulation features of children's touchscreen gestures. In Proceedings of the International Conference on
Multimodal Interaction (ICMI '2016), 333-340. https://doi.org/10.1145/2993148.2993179

50. Joseph W. Sullivan and William Mark. 1991. Intelligent User Interfaces. ACM Press.

51. Jerry R. Thomas. 1980. Acquisition of Motor Skills: Information Processing Differences between Children and Adults. Research Quarterly for Exercise and Sport 51, 1: 158-173. https://doi.org/10.1080/02701367.1980.10609281

52. Elba del Carmen Valderrama Bahamóndez, Thomas Kubitza, Niels Henze, and Albrecht Schmidt. 2013. Analysis of children's handwriting on touchscreen phones. In Proceedings of the International Conference on Human-Computer Interaction with Mobile Devices and Services (MobileHCI'2013), 171-174. https://doi.org/10.1145/2493190.2493222

53. Radu-Daniel Vatavu, Lisa Anthony, and Quincy Brown. 2015. Child or Adult? Inferring Smartphone Users' Age Group from Touch Measurements Alone. In Human-Computer Interaction - INTERACT 2015. Springer International Publishing, 9 pages. https://doi.org/10.1007/978-3-319-22723-8_1

54. Keith Vertanen and Per Ola Kristensson. 2008. On the benefits of confidence visualization in speech recognition. In Proceedings of the ACM SIGCHI Conference on Human Factors in Computing Systems (CHI'2008), 1497-1500. https://doi.org/10.1145/1357054.1357288

55. Stella Vosniadou and William F Brewer. 1992. Mental models of the earth: A study of conceptual change in childhood. Cognitive Psychology 24, 4: 535-585. https://doi.org/10.1016/0010-0285(92)90018-W

56. Greg Walsh. 2011. Distributed participatory design. In Extended Abstracts of the ACM SIGCHI Conference on Human Factors in Computing Systems (CHI'2011), 1061-1064. https://doi.org/10.1145/1979742.1979696

57. Greg Walsh, Alison Druin, Mona Leigh Guha, Elizabeth Foss, Evan Golub, Leshell Hatley, Elizabeth Bonsignore, and Sonia Franckel. 2010. Layered elaboration: a new technique for co-design with children. In Proceedings of the ACM SIGCHI Conference on Human Factors in Computing Systems (CHI'2010), 1237-1240. https://doi.org/10.1145/1753326.1753512

58. Greg Walsh, Elizabeth Foss, Jason Yip, and Allison Druin. 2013. FACIT PD: A Framework for Analysis and Creation of Intergenerational Techniques for Participatory Design. In Proceedings of the ACM SIGCHI Conference on Human Factors in Computing Systems (CHI'2013), 2893-2902. https://doi.org/10.1145/2470654.2481400

59. Jieyu Wang and Anita Komlodi. 2012. Children's formal and informal definition of technology. In 
Proceedings of the iConference (iConference'2012), 587-588. https://doi.org/10.1145/2132176.2132299

60. Julia Woodward, Lisa Anthony, Germaine Irwin, Alex Shaw, Annie Luc, Brittany Craig, Juthika Das, Phillip Hall, Akshay Holla, Danielle Sikich, and Quincy Brown. 2016. Characterizing How Interface Complexity Affects Children's Touchscreen Interactions. In Proceedings of the ACM SIGCHI Conference on Human Factors in Computing Systems (CHI'2016), 1921-1933.

https://doi.org/10.1145/2858036.2858200

61. Robert K. Yin. 2013. Validity and generalization in future case study evaluations. Evaluation 19, 3: 321332. https://doi.org/10.1177/1356389013497081

62. Jason C. Yip, Kiley Sobel, Caroline Pitt, Kung Jin Lee, Sijin Chen, Kari Nasu, and Laura R. Pina. 2017. Examining Adult-Child Interactions in Intergenerational Participatory Design. In Proceedings of the ACM SIGCHI Conference on Human Factors in Computing Systems (CHI'2017), 5742-5754. https://doi.org/10.1145/3025453.3025787

63. Wenxuan Zhou, Jason Croft, Bingzhe Liu, and Matthew Caesar. 2017. NEAt: Network Error AutoCorrect. In Proceedings of the ACM Symposium on SDN Research (SOSR '2017), 157-163. https://doi.org/10.1145/3050220.3050238

64. Cortana | Your Intelligent Virtual \& Personal Assistant. Microsoft. Retrieved September 15, 2017 from https://www.microsoft.com/en-us/windows/cortana
65. Echo \& Alexa - Amazon Devices - Amazon Official Site. Amazon. Retrieved September 15, 2017 from https://www.amazon.com/Amazon-Echo-And-AlexaDevices $/ \mathrm{b}$ ? $\mathrm{ie}=\mathrm{UTF} 8 \&$ node $=9818047011$

66. iOS - Siri - Apple. Apple. Retrieved September 15, 2017 from https:/www.apple.com/ios/siri/

67. OK Google. Google. Retrieved September 15, 2017 from http://ok-google.io/

68. RealtimeBoard | Virtual Whiteboard \& Remote Collaboration tool. Retrieved September 11, 2017 from https://realtimeboard.com/

69. Monster Messenger. Retrieved September 18, 2017 from https://monster-messenger.com/

70. ChatterPix Kids - by Duck Duck Moose. Apple App Store. Retrieved September 15, 2017 from https://itunes.apple.com/us/app/chatterpix-kids-byduck-duck-moose/id734046126? $\mathrm{mt}=8$

71. 2010. FAQ 15: What are the best ways to interview children? LSE Media and Communications. Retrieved September 12, 2017 from http://www.lse.ac.uk/media@1se/research/EUKidsOnli ne/BestPracticeGuide/FAQ/FAQ-15.pdf

72. 2017. The Common Sense Census: Media Use by Kids Age Zero to Eight 2017. Common Sense Media. Retrieved December 10, 2017 from https:/www.commonsensemedia.org/research/thecommon-sense-census-media-use-by-kids-age-zero-toeight-2017 